Classification

Physics Abstracts

$07.60 \mathrm{~F}-64.70 \mathrm{~J}-68.10-82.70$

\title{
Measurement of the rigidity coefficient of a surfactant layer and structure of the oil or water microemulsion interface
}

\author{
J. Meunier \\ Laboratoire de Spectroscopie Hertzienne de l'E.N.S., 24, rue Lhomond, \\ 75231 Paris Cedex 05, France
}

(Reçu le 28 juin 1985, accepté le 16 septembre 1985)

\begin{abstract}
Résumé. - Nous déduisons des mesures ellipsométriques le coefficient de rigidité d'une couche monomoléculaire de surfactant à une interface eau-huile. La monocouche contenant de l'alcool, nous trouvons une valeur très faible du coefficient $K\left(K \approx k_{\mathrm{B}} T\right)$. Nous étudions ensuite les interfaces microémulsion-huile et microémulsion-eau recouvertes du même film. Nous trouvons qu'il existe une zone perturbée dans la microémulsion au contact de cette monocouche, zone dont l'épaisseur est comparable à la taille des objets dans la microémulsion. Les résultats expérimentaux pour les interfaces de très faibles tensions interfaciales suggèrent que celles-ci peuvent être décrites par le modèle d'interfaces ouvertes récemment proposées.
\end{abstract}

\begin{abstract}
We first deduce the rigidity coefficient $K$ of a surfactant monolayer at the oil water interface from ellipsometric measurements. For our surfactant monolayer containing alcohol, we find unusually small values of $K\left(K \approx k_{\mathrm{B}} T\right)$. We then study the oil microemulsion and water microemulsion interfaces, covered with the same film. On the microemulsion side of the film we find a perturbed zone, the thickness of which is comparable with the size of the structure in the bulk. The experimental results suggest that at ultralow surface tension, the recently proposed picture of an open interface, is quite valid.
\end{abstract}

\section{Introduction.}

A five component system : oil, water, salt, surfactant and co-surfactant has a complex phase diagram. At a fixed temperature we may obtain the following sequence of phase equilibria upon increasing salinity $S$ [1] (Fig. 1a).

- $S<S_{1}: 2$ phase region with a lower microemulsion coexisting of an upper organic phase (Winsor I domain). The interface will be denoted by $O M$.

- $S_{1}<S<S_{2}: 3$ phase region with a coexisting lower aqueous phase, a median microemulsion phase and an upper organic phase (Winsor III domain). The two interfaces will be denoted by $\mathrm{OM}$ and WM respectively.

- $S>S_{2}: 2$ phase region with a coexisting lower aqueous phase and an upper microemulsion phase (Winsor II domain). The interface will be denoted by WM. 


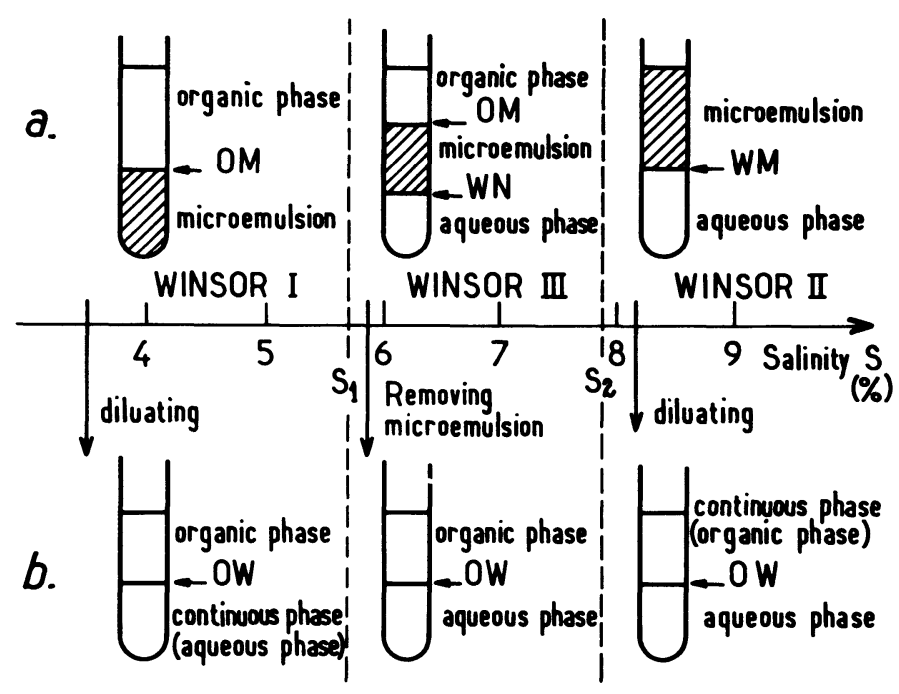

Fig. 1. - The phase equilibrium versus the salinity and the different studied interfaces.

It is now clear [2-5] that microemulsions below $S_{1}$ (respectively above $S_{2}$ ) are a dispersion of oil (or water) droplets in a continuous phase, aqueous or organic respectively. These droplets are surrounded by a layer containing surfactant and co-surfactant molecules. This structure explains that the microemulsion can be diluted by a procedure which allows the droplet density to change without changing the droplet size by adding a continuous phase $[3,6]$ (Fig. 1b). The interface obtained by mixing the continuous phase and the organic phase in the Winsor I domain or the aqueous phase in the Winsor II domain will be denoted by $\mathrm{OW}$.

The middle phase, or microemulsion in the intermediate salinity range $\left(S_{1}<S<S_{2}\right)$, is described by a bicontinuous structure of aqueous and organic phases separated by a mixed surfactant and co-surfactant layer $[2,5,7]$. In this case no dilution procedure is allowed. Thus in the Winsor III domain, the interface obtained upon removing the microemulsion phase will also be denoted by OW (Fig. 1b).

In the non-dilutable systems, the low surface tensions $\gamma$ of the different interfaces have been measured in our laboratory by the technique of surface light scattering [8]. $\gamma_{O M}$ decreases with $S$ while $\gamma_{\mathrm{WM}}$ increases. At a salinity $S=S$ * which is roughly the midpoint salinity of the Winsor III domain, $\gamma_{O M}=\gamma_{W M}=\gamma^{*}$. In the Winsor I and II domains, the measured interfacial tensions have been found to be independent of the droplet density in the microemulsion phase and equal to $\gamma_{\text {ow }}[8,9]$. In the Winsor III domain, the higher surface tension $\left(\gamma>\gamma^{*}\right)$ of the two interfaces is equal to $\gamma_{\text {ow }}[8]$.

The conclusion of this study was that the origin of the low tensions in this case, is the high surface pressure of the monolayer at the interface containing surfactant and co-surfactant molecules. The lower surface tensions $\left(\gamma<\gamma^{*}\right)$ in the Winsor III domain have been explained by the proximity of the critical points $[10,11]$.

In order to describe interfaces in such systems, it is now well accepted that an important supplementary parameter (apart from the interfacial tension) has to be introduced, i.e. the rigidity constant $K$ of the surfactant layer. This parameter is probably also of fundamental interest for the understanding of microemulsion structures [12]. In the following we will present experimental results obtained by ellipsometry, from which we have deduced this rigidity constant for a planar monolayer of surfactant and co-surfactant. We will furthermore present some extra information 
about the structure of the OM and WM interfaces. Though our ellipticity measurements are similar (but more complete) to previous ones on these microemulsions systems [13], some differences will be discussed later.

\section{The ellipticity of an interface.}

The measured ellipticity $\bar{\rho}$ of an interface is given by :

$$
\bar{\rho}=\frac{\pi}{\lambda} \frac{\sqrt{\varepsilon_{1}+\varepsilon_{2}}}{\varepsilon_{1}-\varepsilon_{2}} \eta
$$

where $\lambda$ is the wavelength of the light, $\varepsilon_{1}$ and $\varepsilon_{2}$ the dielectric constants of the two phases; $\eta$ depends upon the interfacial properties and is the sum of two terms :

$$
\eta=\eta^{R}+\eta^{l}
$$

For a planar and flat layer $\eta^{R}=0$, and $\eta^{l}$ is given by Drude's formula [14] to first order in $l / \lambda$ ( $l$ is the thickness of the interface, $\lambda$ the wavelength of the light) :

$$
\eta^{l}=\int \frac{\left(\varepsilon-\varepsilon_{1}\right)\left(\varepsilon-\varepsilon_{2}\right)}{\varepsilon} \mathrm{d} z,
$$

$\varepsilon(z)$ is the dielectric constant through the interface. In the case of a thin but rough interface $\eta^{l}=0$ and $\eta^{R}$ are always negative $[15,16]$ :

$$
\eta^{R}=-\frac{3}{4} \frac{k_{\mathrm{B}} T}{\pi \gamma} \frac{\left(\varepsilon_{1}-\varepsilon_{2}\right)^{2}}{\varepsilon_{1}+\varepsilon_{2}} q_{\mathrm{m}},
$$

$q_{\mathrm{m}}$ is an upper cut-off for the capillary waves. This cut-off is the consequence of the curvature energy of the interfacial layer. The curvature energy $\mathrm{Kq}^{4}$ must be introduced in the calculation of the mean square amplitude $\left\langle\zeta_{q}^{2}\right\rangle$ of a $q$ wave vector fluctuation for an interface in thermal equilibrium (Eq. (5.14) in the paper by Zielinska et al. [16]) :

$$
\left\langle\zeta_{q}^{2}\right\rangle=S_{\mathrm{eq}}(q)=\frac{k_{\mathrm{B}} T}{\Delta \rho . g+\gamma q^{2}+K q^{4}},
$$

$\Delta \rho . g$ is the energy due to gravity, $\Delta \rho$ the density difference between the two phases, $\gamma q^{2}$ the capillary energy and $K q^{4}$ the curvature energy; $K$ is the rigidity coefficient. Substituting this expression into the integrals $(5.16)$ of reference [16], we obtain the value of the cut-off :

$$
q_{\mathrm{m}}=\frac{\pi}{2} \sqrt{\frac{\gamma}{K}} .
$$

We shall first study the OW interfaces and show that this low surface tension interface is described by a thin monolayer $\left(\eta^{R} \gg \eta^{l}\right)$. Thus the measurement of $\eta \sim \eta^{R}$ allows us to obtain the rigidity coefficient $K$ (Sect. 3.1). In a second part we shall study the OM and WM interfaces. In the case $\gamma>\gamma^{*}$ using the $K$ value measured previously, it will now be possible to evaluate the respective contributions of $\eta^{R}$ (roughness) and $\eta^{l}$ (monolayer thickness). These two contributions will not be sufficient to explain the experimental $\eta$ values. We shall therefore introduce a third contribution, $\eta^{L}$, the origin of which is due to the structure of the microemulsion in the vicinity of the monolayer. Furthermore, we shall show that the experimental values of $\eta$, for interfaces where $\gamma<\gamma^{*}$ are in good agreement with the picture of open interfaces [17] (Sect. 3.2). 


\section{Sample preparation and experimental results.}

We studied the same mixtures as those used for surface tension measurements $[8,9]$ : water + sodium chloride (46.8 wt \%), toluene $(47.25 \%)$, butanol $1(3.96 \%)$ and sodium dodecyl sulfate $(1.99 \%)$. In spite of the fact that the origin and the purity of the components and the temperature $\left(20^{\circ} \pm 0.1^{\circ} \mathrm{C}\right)$ were the same, the limiting salinities $S_{1}$ and $S_{2}$ were 5.7 and 7.85 instead of 5.35 and 7.4.

The refractive indices were determined by an Abbe refractometer. The interfacial ellipticity measurements were performed with a home made ellipsometer. Its configuration is based upon that of Jasperson and Schnatterly [18] and mounted vertically to reflect off the horizontal liquid surface.

3.1 Organic phase-Aqueous phase interface (OW). - Figure 2 shows the experimental values of $\eta_{\text {ow }}$ [19] ( $\eta$ at the $O W$ interfaces). $\eta_{\text {ow }}$ is negative and it is possible to compare its variations with respect to $S$ with the variation of $\gamma$ with respect to $S$. We found $\eta_{\text {ow }} \sim-1 / \sqrt{\gamma}$. This result suggests (see Eqs. (5) and (3)) that in this case we have $\eta_{\text {ow }}^{R} \gg \eta_{\text {ow }}^{l}$. This is in good agreement with the previous picture of the interface deduced from surface tension measurements [8] i.e. a surfactant monolayer at the interface. $\eta_{\text {ow }}^{l}$ was calculated using equation (2), taking for the integration limit the molecular length of the surfactant $(l \sim 20 \AA)$ and $\varepsilon=2$. We found $\eta_{\text {ow }}^{l} \approx 0.4$. We then deduce the rigidity coefficient $K$ (Fig. 3a) using $\eta_{\mathrm{ow}}^{R}=\eta_{\mathrm{ow}}-\eta_{\mathrm{ow}}^{l}$, the equations (3) and (5) and the surface tension measurements [8], taking into account the shift of the salinities $S_{1}$ and $S_{2}$ [20]. We found $K \approx k_{\mathrm{B}} T$ and that it decreases slightly with the salinity. This variation is probably due to the screening of the electrostatic forces between the polar heads of the surfactant inside the interfacial monolayer. The monolayer we observe has no curvature, thus it is obviously not exactly the same as the one inside the microemulsion. However, we can expect that these two layers are similar, especially in the Winsor III domain, where the radius of curvature of the monolayer inside the microemulsion is large. Furthermore, the $K$ values obtained are in good agreement with the ones expected for microemulsions [12] and so confirm this idea.

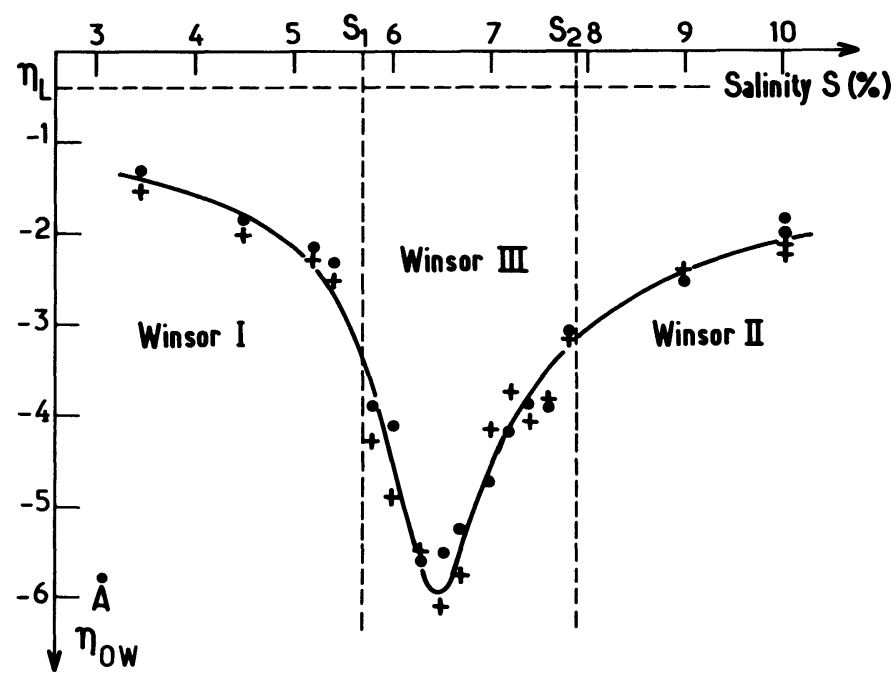

Fig. 2. $-\eta_{\mathrm{ow}}$ at the aqueous-organic phases interfaces. (+) light incident from the organic phase; (0) light incident from the aqueous phase. 

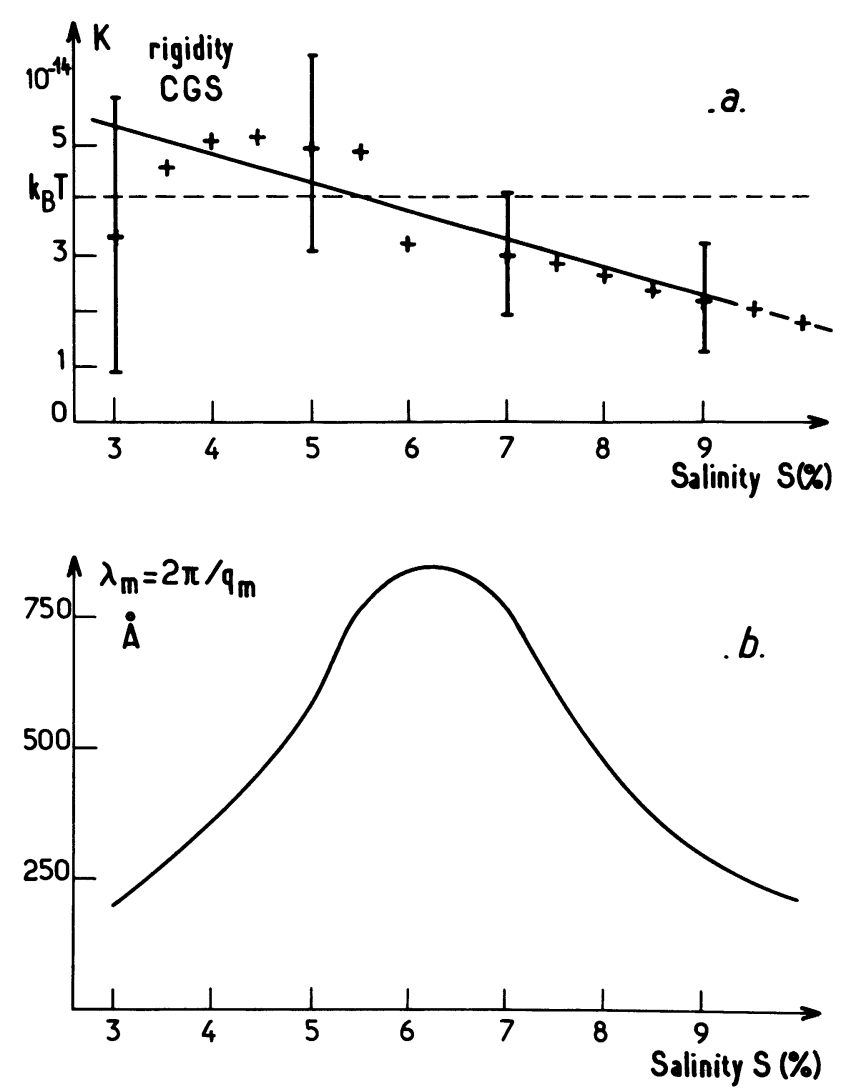

Fig. 3. - a) The rigidity coefficient $K$ of the monolayer interface versus the salinity. b) The wavelength cut-off $\lambda_{\mathrm{m}}$ versus salinity.

From the $K$ value we can deduce the wavelength cut-off $\lambda_{\mathrm{m}}=2 \pi / q_{\mathrm{m}}$ (Fig. 3b). Its large value explains that roughness is not observed on the scale of the microstructures inside the microemulsions [5]. The variation of $\lambda_{\mathrm{m}}$ with $S$ we obtain is the same as that of the structure in the bulk of the microemulsion [11,5] as predicted [12] (see Fig. 6 the radii of the droplets and the persistence length $\xi_{K}$ in appendice I).

3.2 Microemulsion-Organic (OR AQueOus) PhaSe INTERfaces. - Figure 4 shows the experimental values of $\eta_{\mathrm{MO}}$ and $\eta_{\mathrm{MW}}$. There are two small differences with the results of Beaglehole et al. [13]. 1) Our positive peak values of $\eta$ are twice as big. This is probably because we have studied a greater number of salinities in the vicinity of the sharp peaks of the curves. 2) We do not find any change in the slopes of the negative parts of the curves approaching the limiting salinity range in the Winsor III domain. The origin of these slight discrepancies is probably because the experiments performed by Beaglehole et al. have not reached equilibrium in the vicinity of the limiting salinities.

3.2.1 The OM and WM interfaces for $\gamma>\gamma^{*}$. — Let us now assume as has Pouchelon [8, 9], that the surfactant monolayer at these interfaces is the same as the one at the OW interfaces studied in the previous section. It is now possible to evaluate two contributions to $\eta$ i.e. $\eta^{R}$ and $\eta^{l}$. The origin of $\eta^{R}$ is the roughness and is calculated from the experimental values of figure 2 using equations (3) and (5). The only contribution to $\eta^{l}$ is due to the surfactant monolayer thick- 


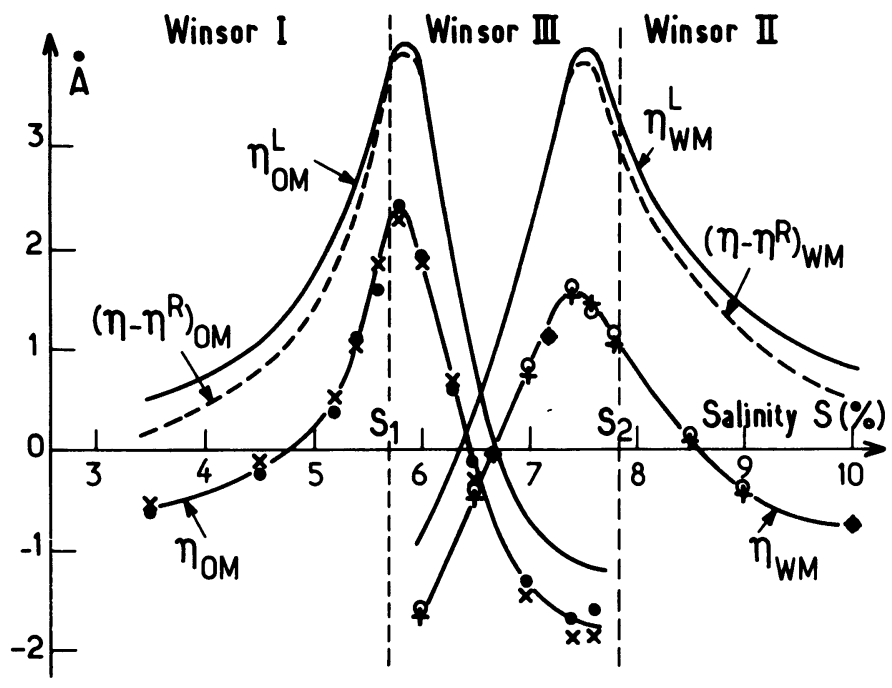

Fig. 4. $-\eta_{\mathrm{ow}}$ at the organic phase-microemulsion interface $:(x)$ light incident from the organic phase ; (๑) light incident from the microemulsion phase. $\eta_{\mathrm{WM}}$ at the aqueous phase microemulsion interface ; (O) light incident from the microemulsion phase; $(+)$ light incident from the aqueous phase. $\left(\eta-\eta^{R}\right)_{\mathrm{OM}}$, $\left(\eta-\eta^{R}\right)_{\mathrm{WM}}$ (dashed lines); $\eta_{\mathrm{OM}}^{L}$ and $\eta_{\mathrm{WM}}^{L}$ (full lines) deduced from the experimental results $\eta_{\mathrm{OM}}$ and $\eta_{\mathrm{WM}}$.

ness and is calculated using equation (2). $\eta^{l}$ is small in the Winsor III domain where it has been neglected. $\eta^{L}=\eta-\left(\eta^{R}+\eta^{l}\right)$, deduced from the experimental results, is not zero, so we have plotted it in figure 4 (we have also plotted $\eta-\eta^{R}$ in the same figure) for the two interfaces OM and WM. $\eta^{L}$ is a positive third contribution to $\eta$. This positive value arises only if the interfacial dielectric constant is larger or smaller than $\varepsilon_{1}$ and $\varepsilon_{2}$ (Eq. (2)) the origin of which might be explained by the following :

- In the Winsor I (or II) domain, microemulsions are droplets of oil (or water) in the continuous phases with hard sphere interactions $[10,11]$. The finite radius, $R$, of the droplets prevents them from nearing the monolayer interface any closer than $R$ [21] (Fig. 5a). Figure 6 shows the variation of $L$ with $S$ deduced from $\eta$ and the radii $R$ of the droplets in the Winsor I and II domains [10,11]. $L$ and $R$ are in good agreement.

- In the Winsor III domain we expect a similar picture i.e. the object in the bulk stays at a distance $L$ away from the surface monolayer (Fig. $5 \mathrm{~b}$ ). This distance is reported in figure 6 . At the limiting salinities we obtain $L \approx 240 \AA$ which is close to the persistence length in the bulk $\xi_{K}=(200 \pm 50) \AA$ (see appendix I) as we expect. In the middle of the Winsor III domain $L$ decreases sharply and reaches an almost zero value at the midpoint salinity, probably because attractive forces between the interfacial monolayer and the objects in the bulk increase quickly. The picture of an excluded volume by steric repulsions is no longer valid.

3.2.2 The OM and WM interfaces for $\gamma<\gamma^{*}$. - What happens on each side of the midpoint salinity for these interfaces ? The $\eta^{L}$ values become negative (Fig. 4), which means that the dielectric constant $\varepsilon$ in the interfacial layer lies between $\varepsilon_{1}$ and $\varepsilon_{2}$. This arises if the interfacial structure changes from a closed to an open interface [17] when $L$ crosses over zero (Fig. $5 \mathrm{c}$ and 5d). The measured $\eta$ is now the sum of two negative values $\eta^{L}$ and $\eta^{R}$. It is difficult to evaluate $\eta^{R}$ for this interfacial structure because the rigidity coefficient is not known. Nevertheless, the monolayer in the bulk is not very different from that of the flat monolayer as previously suggested. 


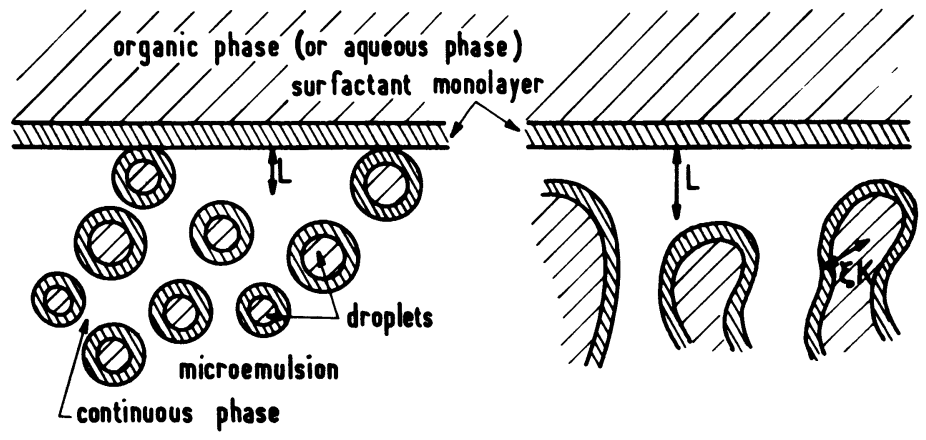

a) The Winsor I or II domain

b) Winsor III domain

$$
\gamma>\gamma *
$$

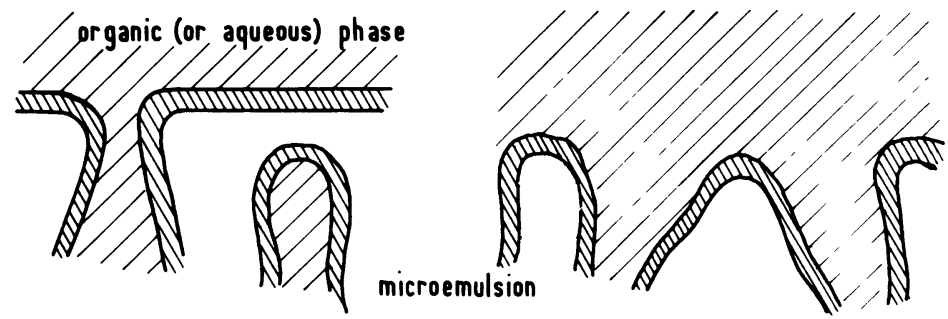
c) The Winsor III domain
$\gamma \sim \gamma *$
d) The Winsor III domain
$\gamma<\gamma$

Fig. 5. - The structure of the interface and of the microemulsion versus the salinity $S$. The interface is passing gradually from a closed interface ( $a$ and $b$ ) to an open interface (c and $d$ ).

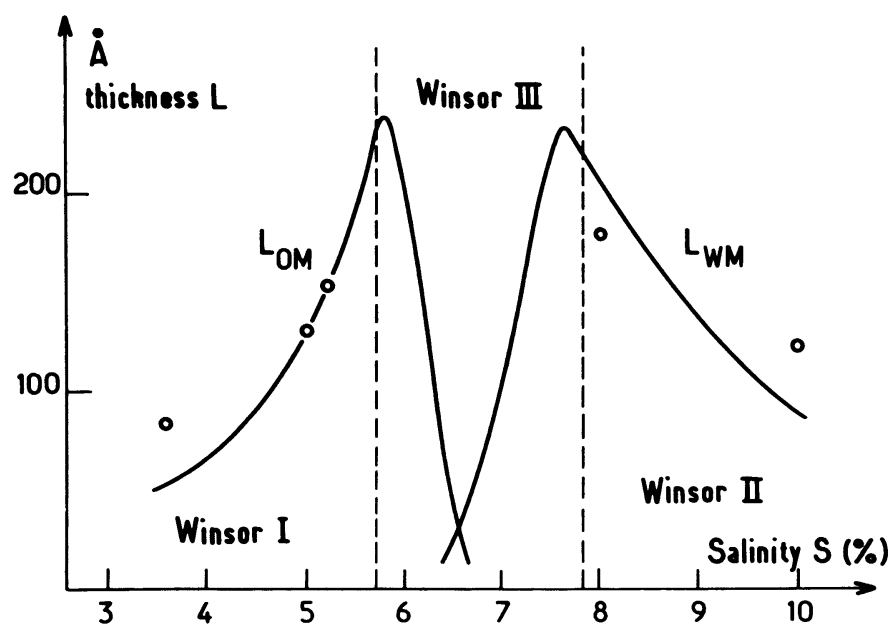

Fig. 6. - The thickness $L$ versus the salinity (full line) and the radii of the micelles in the Winsor I and II domain (open circles). 
Consequently we have kept the same $K$ value, and, using the critical behaviour of the interface approaching the limiting salinities, we have obtained constant values $\eta_{\mathrm{OM}}^{R} \approx 0.61 \AA$ and $\eta_{\mathrm{WM}}^{R} \approx 0.66 \AA$ (see appendix II).

In the vicinity of $S_{1}$ (or $S_{2}$ ) each mean field theory gives the same profile of $\Delta \rho$ or $\Delta n$ versus $z$ through the interface [24]. (This mean field profile is close to the profile given by other theories [25]) :

$$
\Delta n=\frac{1}{2}\left(n_{2}-n_{1}\right) \tanh (2 z / L)
$$

where $L$ is the thickness of the interface. By combining equations (2) and (6) we can calculate $\eta^{L}$ :

$$
\eta^{L} \approx-\Delta n^{2} L \quad\left(\text { with } \Delta n=n_{2}-n_{1}\right) \text {. }
$$

Using scaling laws for $\Delta n$ (see appendix II) and $L \approx L_{0} \Delta \rho^{-2}$ one obtains :

$$
\eta^{L} \approx-n_{0}^{2} L_{0} \quad\left(n_{0} \text { is given in appendix II }\right)
$$

which is a constant. So, in the vicinity of limiting salinities we obtain $\eta=\eta^{L}+\eta^{R}$ which is also constant.

- This picture is in good agreement with the experimental results of $\eta_{O M}$ in the vicinity of $S_{2}$ (Fig. 4). For $S_{2}=7.6$ we find $L_{\mathrm{OM}} \approx 1150 \AA$.

- In the vicinity of $S_{1}$ we find smaller $L$ values $\left(L_{\mathrm{wM}}=340 \AA\right.$ for $\left.S=6\right)$ which are not much greater than $\xi_{K}=240 \AA$ in the bulk. This explains why the experimental values of $\eta_{\mathrm{WM}}$ in the vicinity of $S_{1}$ are not constant. Our experimental values stay much further from the critical end point.

The experimental results we thus obtain are quite consistent with the hypothesis that the rigidity constant is the same as the one for the previous interfaces.

\section{Conclusion.}

Our interpretation of the ellipticity of the OW, OM and WM interfaces is very different from that of Beaglehole et al. who explain their data in terms of wetting phenomena.

The ellipsometric technique is sensitive to the mean slope of the interfacial roughness and is consequently very sensitive to the cut-off wavelength of the roughness. The origin of the cut-off on a layer is the rigidity $K$ and the cut-off measurement is a means of measuring $K$. We have obtained this $K$ coefficient of a surfactant monolayer of low surface tension, the roughness of which is the main reason for the interface ellipticity. Our $K$ experimental values are larger but comparable with those measured on lamellar systems which are close to a microemulsion [26, 27]. Then, knowing the rigidity of the monolayer, we were able to analyse the structure of the OM and WM interfaces : away from the $\gamma^{*}$, the steric repulsion hinders the approach of the objects in the microemulsion (droplets in the Winsor I and II domains) to the interfacial monolayer. In the vicinity of $\gamma^{*}$ a large attractive force between the interfacial monolayer and the objects in the bulk must exist. We have thus shown that the ellipsometric measurements agree with a model of transition of the interface from a closed to an open interface.

\section{Appendix I.}

The persistence length in the Winsor III domain is given by [22] :

$$
\xi_{K}=\frac{6 \phi_{\mathrm{o}} \phi_{\mathrm{w}}}{\Sigma C_{\mathrm{T}}}
$$


$\phi_{\mathrm{O}}$ and $\phi_{\mathrm{W}}$ are the volume fractions of oil and water in the microemulsion, $C_{\mathrm{T}}$ is the number of surfactant molecules in the unity volume and $\Sigma$ is the area per SDS polar head. At the limiting salinities $S_{1}\left(S_{2}\right)$ we have $\phi_{\mathrm{o}} \approx 0.2$ (or 0.8$), \phi_{\mathrm{W}} \approx 0.8$ (or 0.2 ) [23] and $\Sigma=(61 \pm 8) \AA$ from which we can deduce $\xi_{K}=(200 \pm 50) \AA$.

\section{Appendix II.}

In the Winsor III domain and for $\gamma<\gamma^{*}, \gamma$ and $\Delta n$ have a critical behaviour [11, 23] :

$$
\gamma \approx \gamma_{0}(\Delta \rho)^{4} ; \quad \Delta n \approx n_{0}(\Delta \rho)
$$

one obtains from equations (3) and (5) :

$$
\eta^{R} \approx-\frac{3}{8} k_{\mathrm{B}} T \frac{\left(n_{1}+n_{2}\right)^{2}}{\left(n_{1}^{2}+n_{2}^{2}\right)} \frac{n_{0}^{2}}{\sqrt{\gamma_{0} K}}
$$

$n_{1}, n_{2}$ are the refractive indexes of the two phases and $\Delta n=n_{1}-n_{2}$.

Keeping the $K$ value measured for the interfaces $\gamma>\gamma^{*}$ we obtain constant values $\eta_{\mathrm{OM}}^{R}$ and $\eta_{\mathrm{WM}}^{R}$ in the vicinity of the limiting salinities.

1) $\mathrm{OM}$ interface and $S=7.6: \gamma_{0}=62$ CGS; $n_{0}=0.73$ CGS. We obtain $\eta^{R} \approx 0.61 \AA$.

2) WM interface and $S=6: \gamma_{0}=130 \mathrm{CGS} ; n_{0}=0.95 \mathrm{CGS}$. We obtain $\eta^{R} \approx 0.66 \AA$.

\section{References}

[1] Winsor, P. A., Trans. Faraday Soc. 44 (1948) 376.

[2] Kaler, E. W., Davis, H. T. and Scriven, L. E., J. Chem. Phys. 79 (1983) 5685.

[3] Cazabat, A. M., J. Physique Lett. 44 (1983) 593.

[4] Tabony, J. and De Geyer, A., in Surfactant in Solution, K. L. Mittal and P. Bothorel, eds. (Plenum Press).

[5] Auvray, L., Cotton, J. P., Ober, R., Taupin, C., J. Physique 45 (1984) 913.

[6] GraciaA, A., Lachaise, J., Martinez, A., Bourrel, M. and Chambu, D., C. R. Acad. Sci. B 282 (1976) 547.

[7] SCriven, L., in Micellization, Solubilization and Microemulsions, K. Mittal, ed. (Plenum, N. Y.) 1977, p. 277.

[8] Pouchelon, A., Meunier, J., Langevin, D., Chatenay, D. and Cazabat, A. M., Chem. Phys. Letters 76 (1980) 277.

[9] Pouchelon, A., Chatenay, D., Meunier, J. and Langevin, D., J. Colloid Interface Sci. 42 (1981) 418.

[10] Cazabat, A. M., Langevin, D., Meunier, J. and Pouchelon, A., Adv. Colloid Interface Sci. 16 (1982) 175.

[11] Cazabat, A. M., Langevin, D., Meunier, J. and Pouchelon, A., J. Physique Lett. 43 (1982) L-89.

[12] De Gennes, P. G. and Taupin, C., J. Phys. Chem. 86 (1982) 2294.

[13] Beaglehole, D., Clarkson, M. T. and Upton, A., J. Colloid Interface Sci. 101 (1984) 330.

[14] Drudde, P., Ann. Phys. 43 (1981) 126, and Précis d'Optique (traduction française) t. 2, p. 43.

[15] Beaglehole, D., Physica B 100 (1980) 163.

[16] Zielinka, B. J. A., Bedeaux, D. and Vlieger, J., Physica A 107 (1981) 91.

[17] Auvray, L., J. Physique Lett. 46 (1985) L-163.

[18] JaSPerson, S. N. and Schartterly, S. E., Rev. Sci. Instrum 40 (1969) 761.

[19] In the Winsor I and II domain, we don't use exactly the continuous phase, but a very diluted microemulsion (10 to 15 times) in order to avoid some difficulties described by POUCHELON [9]. 
[20] Pouchelon, A. et al., found $S^{*}=6.35$. In our experiments we have taken $S^{*}=6.5$, the salinity of the maximum of the curve $\eta_{\mathrm{ow}}$ versus $S$.

[21] Asakura, S. and Oosawa, F., J. Chem. Phys. 22 (1954) 1255.

[22] Jouffroy, J., Levinson, P. and De Gennes, P. G., J. Physique 43 (1982) 1241.

[23] Pouchelon, A., thèse de Docteur-Ingénieur, Paris 1982, p. 18.

[24] Talmon, Y., Prager, S., J. Chem. Phys. 69 (1978) 2984.

[25] Huang, J. S. and Webb, W. W., J. Chem. Phys. 50 (1969) 3677.

[26] Di Meglio, J. M., Dvolaitzky, M. and Taupin, C., J. Phys. Chem. 89 (1985) 871.

[27] Di Meglio, J. M., Dvolaitzky, M., Léger, L. and Taupin, C., Phys. Rev. Lett. 54 (1985) 1686. 